\title{
Universality of phase diagrams in QCD and QCD-like theories
}

\section{Masanori Hanada ${ }^{\dagger}$}

Department of Physics, University of Washington, Seattle, WA 98195-1560, USA

E-mail: mhanada@u.washington.edu

\section{Naoki Yamamoto ${ }^{* \dagger}$}

Institute for Nuclear Theory, University of Washington, Seattle, WA 98195-1550, USA

E-mail: nyama@u.washington.edu

\begin{abstract}
We show the universality of phase diagrams in QCD and QCD-like theories through the large- $N_{c}$ equivalence. The whole phase diagrams are identical between $\mathrm{QCD}$ at finite isospin chemical potential and $\mathrm{SO}\left(2 N_{c}\right)$ and $\mathrm{Sp}\left(2 N_{c}\right)$ gauge theories at finite baryon chemical potential. In the chiral limit, they are also identical to that of QCD at finite chiral chemical potential. Outside the pion or diquark condensed phase in these theories, the universality of phase diagrams is applicable to QCD at finite baryon chemical potential. We further argue that the universality may work approximately even for $N_{c}=3$. Our result makes it possible to study QCD at finite baryon chemical potential and high temperature, especially the chiral phase transition, using sign-free theories on the lattice.
\end{abstract}

XXIX International Symposium on Lattice Field Theory

July 10-16 2011

Squaw Valley, Lake Tahoe, California

*Speaker.

${ }^{\dagger}$ Supported by JSPS. 


\section{Introduction}

One of the most important questions within the standard model is to unravel the phases of QCD. In spite of its various phenomenological significance, understanding of the properties of QCD at finite baryon chemical potential $\mu_{B}$ has been hampered mainly because of the sign problem: the fermion determinant in the QCD action is no longer real and positive at $\mu_{B} \neq 0$ so that the conventional Monte Carlo technique fails. Fortunately, there are class of theories which may resemble $\mathrm{QCD}$ at $\mu_{B} \neq 0$ but have no sign problem even at nonzero chemical potential. Such examples include QCD with isospin chemical potential $\mu_{I}$ [1], two-color QCD with even degenerate flavors $N_{f}$ at $\mu_{B} \neq 0$ [2, 3], QCD with fermions in the adjoint representation at $\mu_{B} \neq 0$ [3], $\mathrm{SO}\left(2 N_{c}\right)$ gauge theory at $\mu_{B} \neq 0$ [4, 5, 6] (see $\operatorname{Sec} .2$ ), and $\operatorname{Sp}\left(2 N_{c}\right)$ gauge theory with even degenerate flavors $N_{f}$ at $\mu_{B} \neq 0$ [6]. Although one can study the properties of these sign-free theories on the lattice, it is a priori unclear how and if these theories capture the physics of real QCD at $\mu_{B} \neq 0$.

Recently it has been shown by the present authors [6] that the whole or the part of phase diagrams of these theories are universal if one takes the limit of large number of colors $N_{c}$. The relations are summarized in Fig. 1 (see Sec. 3). An equivalence between $\mathrm{SO}\left(2 N_{c}\right)$ gauge theory at $\mu_{B} \neq 0$ and QCD at $\mu_{B} \neq 0$ was first pointed out in [4] and was further investigated in [5]. The universality of phase diagrams can also be extended (for even $N_{f}$ in the chiral limit) to the other sign-free theory, QCD at nonzero chiral chemical potential $\mu_{5}$ (see Sec. 4), which is of some interest in relation to the chiral magnetic effect [7]. From this universality, one can learn QCD phase diagram at $\mu_{B} \neq 0$ by using sign-free QCD at $\mu_{I} \neq 0$ and $\operatorname{SO}\left(2 N_{c}\right)$ and $\operatorname{Sp}\left(2 N_{c}\right)$ gauge theories at $\mu_{B} \neq 0$ in the large- $N_{c}$ limit, and hopefully, for $N_{c}=3$. There are actually evidences that the universality is valid approximately even in three-color QCD (see Sec. 5).

\section{Phase diagram of QCD-like theories: an example of $\mathbf{S O}\left(2 N_{c}\right)$ gauge theory}

The Lagrangian of the gauge theories in the Euclidean spacetime is given by

$$
\mathscr{L}_{G}=\frac{1}{4 g_{G}^{2}} \operatorname{Tr}\left(F_{\mu \nu}^{G}\right)^{2}+\sum_{f=1}^{N_{f}} \bar{\psi}_{f}^{G}(\mathscr{D}+m) \psi_{f}^{G},
$$

where $G$ denotes the gauge group $\mathrm{SU}\left(N_{c}\right), \mathrm{SO}\left(2 N_{c}\right)$, or $\mathrm{Sp}\left(2 N_{c}\right)$ and $f$ denotes the flavor index. $F_{\mu \nu}^{G}$ is the field strength of each gauge field $A_{\mu}^{G}=A_{\mu a}^{G} T_{a}^{G}$. The Dirac fermion $\psi_{f}^{G}$ belongs to the fundamental representation of the gauge group $G$ and we take the degenerate quark mass $m_{f}=m$ for simplicity. The Dirac operator $\mathscr{D}$ is defined as $\mathscr{D}=\gamma^{\mu} D_{\mu}+\mu \gamma^{4}$ with quark chemical potential $\mu$, and $\mathscr{D}=\gamma^{\mu} D_{\mu}+\frac{1}{2} \mu_{I} \gamma^{4} \tau^{3}$ with isospin chemical potential $\mu_{I}=2 \mu$ for even $N_{f}$.

As an example, let us consider the properties of $\mathrm{SO}\left(2 N_{c}\right)$ gauge theory at $\mu_{B} \equiv 2 N_{c} \mu \neq 0$. From $\left(A_{\mu}^{\mathrm{SO}}\right)^{*}=-A_{\mu}^{\mathrm{SO}}$, one has the relation:

$$
C \gamma_{5} \mathscr{D}(\mu) C \gamma_{5}=\mathscr{D}(\mu)^{*}
$$

where $C$ is the charge conjugation matrix. From (2.2) and chiral symmetry $\left\{\gamma_{5}, \mathscr{D}\right\}=0$, if $i \lambda_{n}$ is one of the eigenvalues of $\mathscr{D}$, eigenvalues appear in quartet $\left(i \lambda_{n},-i \lambda_{n}, i \lambda_{n}^{*},-i \lambda_{n}^{*}\right){ }^{1}$ Therefore, $\operatorname{det}[\mathscr{D}(\mu)+m] \geq 0$ and the Monte Carlo technique is available at $\mu_{B} \neq 0[4,5,6]$.

${ }^{1}$ Note that, when $\lambda_{n}$ is real or pure imaginary, this quartet reduces to two sets of doublets $\left(i \lambda_{n},-i \lambda_{n}\right)$ with their eigenvectors being linearly independent from the anti-unitary symmetry (2.2). 


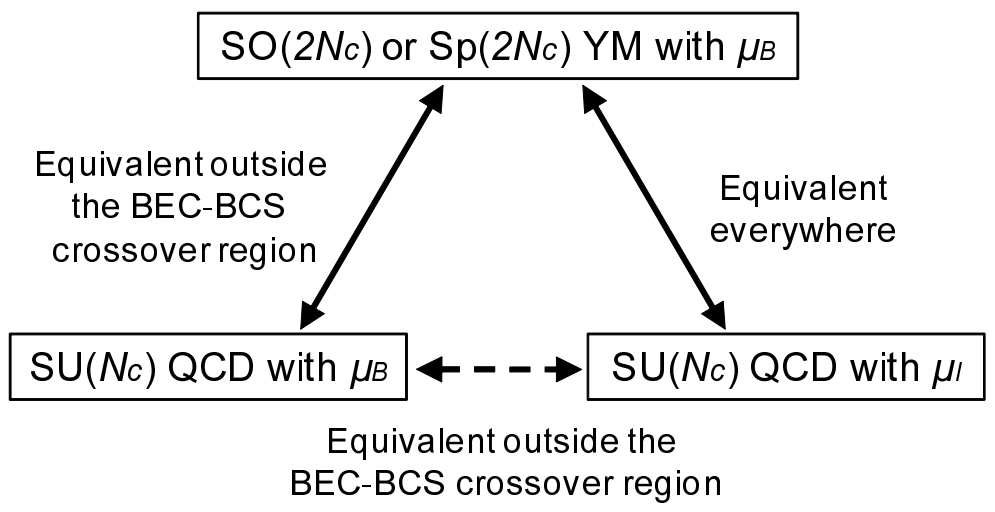

Figure 1: Relations between $\mathrm{SU}\left(N_{c}\right) \mathrm{QCD}$ at $\mu_{B} \neq 0$ and $\mu_{I} \neq 0$ and $\mathrm{SO}\left(2 N_{c}\right)$ and $\operatorname{Sp}\left(2 N_{c}\right)$ Yang-Mills (YM) theories at $\mu_{B} \neq 0$ through the large- $N_{c}$ orbifold equivalence. QCD at $\mu_{I} \neq 0$ can be obtained from $\mathrm{SO}\left(2 N_{c}\right)$ or $\mathrm{Sp}\left(2 N_{c}\right)$ gauge theory at $\mu_{B} \neq 0$ by the orbifold projection in the whole phase diagram, while $\mathrm{QCD}$ at $\mu_{B} \neq 0$ can be obtained outside the BEC-BCS crossover region. As a result, QCD at $\mu_{B} \neq 0$ is equivalent to $\mathrm{QCD}$ at $\mu_{I} \neq 0$ outside the $\mathrm{BEC}-\mathrm{BCS}$ crossover region.

When $m=\mu_{B}=0$, the Lagrangian (2.1) has the enhanced chiral symmetry $\mathrm{U}\left(2 N_{f}\right)$ at the classical level [compared with $\mathrm{SU}\left(N_{f}\right)_{L} \times \mathrm{SU}\left(N_{f}\right)_{R} \times \mathrm{U}(1)_{B} \times \mathrm{U}(1)_{A}$ in the usual three-color QCD] owing to the anti-unitary symmetry (2.2). At the quantum level, $\mathrm{U}(1)_{A} \subset \mathrm{U}\left(2 N_{f}\right)$ is explicitly broken by the axial anomaly and $\mathrm{SU}\left(2 N_{f}\right)$ symmetry remains. One can indeed rewrite the fermionic part of the Lagrangian (2.1) manifestly invariant under $\mathrm{SU}\left(2 N_{f}\right)$, using the new variable $\Psi=\left(\psi_{L}, \sigma_{2} \psi_{R}^{*}\right)^{T}$ :

$$
\mathscr{L}_{\mathrm{f}}=i \Psi^{\dagger} \sigma_{\mu} D_{\mu} \Psi
$$

where $\sigma_{\mu}=\left(-i, \sigma_{k}\right)$ with the Pauli matrices $\sigma_{k}$. The chiral symmetry $\operatorname{SU}\left(2 N_{f}\right)$ is spontaneously broken down to $\mathrm{SO}\left(2 N_{f}\right)$ by the formation of the chiral condensate $\langle\bar{\psi} \psi\rangle$, leading to the $2 N_{f}^{2}+$ $N_{f}-1$ Nambu-Goldstone (NG) bosons living on the coset space $\mathrm{SU}\left(2 N_{f}\right) / \mathrm{SO}\left(2 N_{f}\right)$. In contrast to real QCD, there are not only $\mathrm{U}(1)_{B}$ neutral NG modes with the quantum numbers $\Pi_{a}=\bar{\psi} \gamma_{5} P_{a} \psi$ (just like the usual pions), but also $\mathrm{U}(1)_{B}$ charged $\mathrm{NG}$ modes with the quantum numbers $\Sigma_{S}=$ $\psi^{T} C \gamma_{5} Q_{S} \psi$ and $\Sigma_{S}^{\dagger}=\psi^{\dagger} C \gamma_{5} Q_{S} \psi^{*}$. Here $P_{a}$ are traceless and Hermitian $N_{f} \times N_{f}$ matrices, $P_{a}=P_{a}^{\dagger}$ $\left(a=1,2, \cdots, N_{f}^{2}-1\right)$, and $Q_{S}$ are symmetric $N_{f} \times N_{f}$ matrices, $Q_{S}^{T}=Q_{S}\left(S=1,2, \cdots, N_{f}\left(N_{f}+\right.\right.$ 1)/2), in the flavor space. The chiral perturbation theory describing these NG modes for small $\mu$ is exactly the same as that of $\mathrm{SU}\left(N_{c}\right)$ gauge theory with adjoint fermions $\mu_{B} \neq 0$ considered in [3], because their symmetry breaking patterns are the same [5, 6].

Let us consider the zero-temperature $(T=0)$ ground state of the theory. For small $\mu>m_{\pi} / 2,{ }^{2}$ it is energetically favorable for the $\mathrm{U}(1)_{B}$ charged NG modes $\Sigma_{S}$ with the excitation energy $m_{\pi}-2 \mu$ to form the Bose-Einstein condensation (BEC). On the other hand, at sufficiently large $\mu$, the one-gluon exchange interaction in the $\psi \psi$-channel is attractive in the color symmetric channel. According to the Bardeen-Cooper-Schrieffer (BCS) mechanism, this leads to the condensation of the diquark pairing. Taking into account the Pauli principle, the BCS diquark pairing must be flavor symmetric, and takes the form $\left\langle\psi^{T} C \gamma_{5} Q_{S} \psi\right\rangle \neq 0$. Since this BCS pairing has the same

\footnotetext{
${ }^{2}$ Note that the chiral perturbation theory breaks down when $\mu \sim m_{\rho} / 2$, where $m_{\rho}$ is the mass of the lowest non-NG mode (i.e., $\rho$ meson mass).
} 
$T 4$

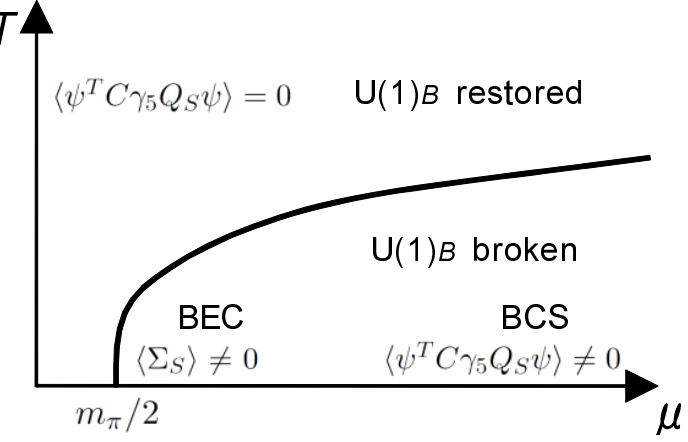

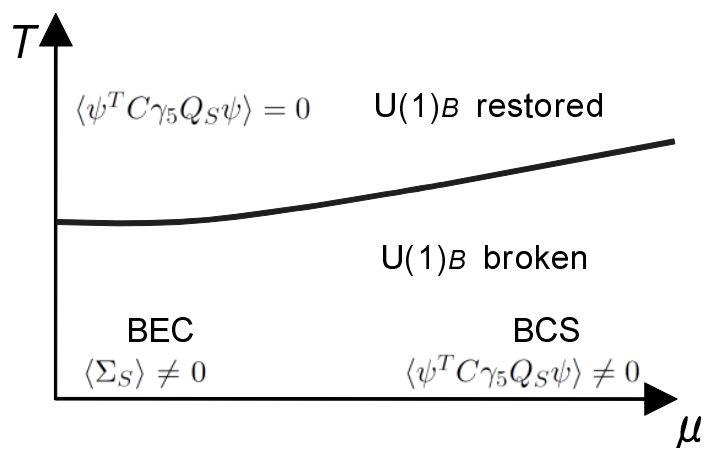

Figure 2: Phase diagram of $\mathrm{SO}\left(2 N_{c}\right)$ gauge theory at $\mu_{B} \neq 0$ for $m>0$ (left) and $m=0$ (right).

quantum numbers and breaks the same symmetry as the $\mathrm{BEC}\left\langle\Sigma_{S}\right\rangle \neq 0$ at small $\mu_{B}$, it is natural to assume no phase transition between the BEC and BCS regions, similarly to the BEC-BCS crossover phenomena in ultracold Fermi gases. The phase diagram of this theory is summarized in Fig. 2 [6].

One can check that phase diagrams of QCD at $\mu_{I} \neq 0[1]$ and $\operatorname{Sp}\left(2 N_{c}\right)$ gauge theory at $\mu_{B} \neq 0$ [6] are qualitatively similar to that of $\mathrm{SO}\left(2 N_{c}\right)$ gauge theory at $\mu_{B} \neq 0$ in Fig. 2] independently of $N_{c} \geq 2$ though the quantum numbers of the condensates are different. More remarkably, one can show that these phase diagrams are universal in the large- $N_{c}$ limit, which we shall argue below in more detail.

\section{Large- $N_{c}$ orbifold equivalence}

The proposed universality can be shown by using the technique of the large- $N_{c}$ orbifold equivalence. The idea of the orbifold equivalence first originates from the string theory [8], and later it is generalized within the quantum field theories without any reference to the string theory.

The main idea is as follows. Suppose that we have some gauge theory. We first choose some discrete symmetry of the theory. Here we call the original theory the parent. Next we eliminate all the degrees of freedom which are not invariant under the discrete symmetry. We call this procedure the projection. As a result of the projection we obtain a new theory, which we call the daughter. Then one can show that a class of correlation functions and observables are equivalent between the parent and daughter theories in the large- $N_{c}$ limit. This is the large- $N_{c}$ orbifold equivalence. The field theoretical proof to the all orders in the perturbation theory was given in [9], and nonperturbative proof in certain gauge theories was given in [10]. There is a caution to use this orbifold equivalence: this equivalence breaks down if the discrete symmetry for the projection is broken spontaneously in the parent theory [11].

As an example, let us consider the projection from $\mathrm{SO}(2 N c)$ gauge theory with fundamental fermions at $\mu_{B} \neq 0$ (parent theory) to QCD at $\mu_{B} \neq 0$ (daughter theory). For an earlier work of the orbifold projection from $\mathrm{SO}\left(2 N_{c}\right)$ to $\mathrm{SU}\left(N_{c}\right)$ gauge theories, see [12]. We choose the projection conditions for the gauge field $A_{\mu}^{\mathrm{SO}}$ and the fermion $\psi_{a}^{\mathrm{SO}}$ as $[4,5,6]$,

$$
A_{\mu}^{\mathrm{SO}}=J_{c} A_{\mu}^{\mathrm{SO}} J_{c}^{-1}, \quad \psi_{a}^{\mathrm{SO}}=\omega\left(J_{c}\right)_{a a^{\prime}} \psi_{a^{\prime}}^{\mathrm{SO}},
$$


where $J_{c}=-i \sigma_{2} \otimes \mathbf{1}_{N_{c}}$ and $\omega=e^{i \pi / 2}$ generate $\mathbb{Z}_{4}$ subgroups of $\operatorname{SO}\left(2 N_{c}\right)$ and $\mathrm{U}(1)_{B} \cdot{ }^{3}$ From these projection conditions, we obtain new gauge field and new fermion field. By a straightforward calculation, one can show that the resulting theory is the $\mathrm{U}\left(N_{c}\right)$ gauge theory (which can be thought as $\mathrm{SU}\left(N_{c}\right)$ gauge theory up to $1 / N_{c}^{2}$ correction at large $N_{c}$ ) with fundamental fermions at $\mu_{B} \neq$ 0 . Then, due to the large- $N_{c}$ orbifold equivalence, a class of order parameters, e.g., the chiral condensate, must be equivalent between above two theories. However, the $\mathbb{Z}_{4}$ discrete symmetry used for the projection of the fermion field, which is a part of $\mathrm{U}(1)_{B}$, is spontaneously broken down to $\mathbb{Z}_{2}$ inside the BEC-BCS crossover region. Therefore, the orbifold equivalence between these two theories is valid outside the BEC-BCS crossover region of $\mathrm{SO}\left(2 N_{c}\right)$ gauge theory at $\mu_{B} \neq 0$.

One can also construct the projection from $\mathrm{SO}\left(2 N_{c}\right)$ gauge theory at $\mu_{B} \neq 0$ to QCD at $\mu_{I} \neq 0$ for even $N_{f}$ by choosing another discrete symmetry [6],

$$
A_{\mu}^{\mathrm{SO}}=J_{c} A_{\mu}^{\mathrm{SO}} J_{c}^{-1}, \quad \psi_{a f}^{\mathrm{SO}}=\left(J_{c}\right)_{a a^{\prime}} \psi_{a^{\prime} f^{\prime}}^{\mathrm{SO}}\left(J_{i}^{-1}\right)_{f^{\prime} f},
$$

where $J_{i}=-i \sigma_{2} \otimes 1_{N_{f} / 2}$ generates $\mathbb{Z}_{4}$ subgroup of $\mathrm{SU}(2)$ isospin symmetry and the projection condition for the gauge field is the same as (3.1). In this case, the isospin symmetry is unbroken including the BEC-BCS crossover region (when we consider the degenerate quark mass) so that the orbifold equivalence holds everywhere in the phase diagram.

By repeating the same argument for $\operatorname{Sp}\left(2 N_{c}\right)$ gauge theory at $\mu_{B} \neq 0$, we obtain the "family tree" of QCD and QCD-like theories as shown in Fig. 1 [6]. In particular, through the equivalence with $\mathrm{SO}\left(2 N_{c}\right)$ or $\mathrm{Sp}\left(2 N_{c}\right)$ gauge theory, we obtain the equivalence between QCD at $\mu_{B} \neq 0$ and $\mathrm{QCD}$ at $\mu_{I} \neq 0$ outside the BEC-BCS crossover region. Since QCD at $\mu_{I} \neq 0$ corresponds to the theory without the complex phase of the fermion determinant of QCD at $\mu_{B} \neq 0$, it follows that the phase-quenched approximation is exact in the large- $N_{c}$ limit there.

\section{QCD at nonzero chiral chemical potential}

One can also show the equivalence of phase diagrams between QCD at $\mu_{5} \neq 0$ and QCD at $\mu_{I} \neq 0$ in the chiral limit for even $N_{f}$. To see this, we first consider the orbifold projections from $\mathrm{SO}\left(2 N_{c}\right)$ gauge theory at $\mu_{5} \neq 0$ to QCD at $\mu_{5} \neq 0$ given by (3.1) and to QCD at nonzero isospinchiral chemical potential $\mu_{I}^{5}$ given by (3.2). Here $\mu_{5}=2 \mu$ corresponds to the quark chemical potential $+\mu$ for $\psi_{R}$ and $-\mu$ for $\psi_{L}$, and $\mu_{I}^{5}=2 \mu$ corresponds to $+\mu$ for $u_{R}$ and $d_{L}$ and $-\mu$ for $u_{L}$ and $d_{R}$ when $N_{f}=2$. We then note that QCD at $\mu_{I}^{5} \neq 0$ is equivalent to QCD at $\mu_{I} \neq 0$ by relabeling $d_{L} \leftrightarrow u_{L}$ in the chiral limit. From the large- $N_{c}$ orbifold equivalence, the whole phase diagram of $\mathrm{QCD}$ at $\mu_{5} \neq 0$ must be thus identical to that of QCD at $\mu_{I} \neq 0$ in the chiral limit [or $\operatorname{SO}\left(2 N_{c}\right)$ gauge theory at $\mu_{B} \neq 0$ in the right panel of Fig. 2 , ${ }^{4}$ where the pion condensate $\left\langle\bar{d} \gamma_{5} u\right\rangle \neq 0$ (or diquark condensate $\left\langle\psi^{T} C \gamma_{5} Q_{S} \psi\right\rangle \neq 0$ ) is replaced by the "chiral condensate" $\left\langle\bar{\psi}_{L} \psi_{R}\right\rangle \neq 0$; the BEC-BCS crossover region of $\left\langle\bar{\psi}_{L} \psi_{R}\right\rangle \neq 0$ appears as a function of $\mu_{5}$. Especially, at sufficiently large $\mu_{5}$, the critical temperature of the chiral phase transition is given by the well-known BCS formula

\footnotetext{
${ }^{3}$ Here $J_{c}$ is chosen such that it satisfies the regularity condition $\operatorname{Tr}\left(J_{c}^{n}\right)=0$ when $J_{c}^{n}$ does not belong to the center of $\mathrm{SO}\left(2 N_{c}\right)$, i.e., $J_{c}^{n} \neq \pm \mathbf{1}_{2 N_{c}}$. This condition is necessary for the proof of the perturbative orbifold equivalence [9].

${ }^{4}$ Note that, in QCD at $\mu_{I} \neq 0$ or $\mathrm{SO}\left(2 N_{c}\right)$ gauge theory at $\mu_{B} \neq 0$ in the chiral limit, there is no chiral condensate but pion or diquark condensate (see, e.g., [13]).
} 
$T_{c}=\left(e^{\gamma} / \pi\right) \Delta$, where $\Delta$ is the fermion gap at large $\mu_{5}$ and $\gamma \approx 0.577$ is the Euler-Mascheroni constant.

It seems that recent model calculations $[14,15,16]$ do not capture the physics from intermediate to large $\mu_{5}$ due to the cutoff of the model. We also note that, once $\mu_{5}>0$ is turned on, $\left\langle\bar{\psi}_{L} \psi_{R}\right\rangle$ and $\left\langle\bar{\psi}_{R} \psi_{L}\right\rangle$ are independent variables unlike QCD at $\mu_{B} \neq 0$ where $\left\langle\bar{\psi}_{L} \psi_{R}\right\rangle=\left\langle\bar{\psi}_{R} \psi_{L}\right\rangle$. Physically, as $\mu_{5}$ increases, $\left\langle\bar{\psi}_{L} \psi_{R}\right\rangle$ should become larger at $T=0$ since the phase space for the pairing near the Fermi surface increases, and so does its critical temperature; the genuine order parameter of chiral symmetry breaking is $\left\langle\bar{\psi}_{L} \psi_{R}\right\rangle$ rather than the conventional $\langle\bar{\psi} \psi\rangle$.

\section{Approximate universality in real QCD}

Now we discuss what to extent the universality is satisfied in three-color QCD. First we can explicitly check the universality at sufficiently large $\mu_{B}$ and $\mu_{I}$ by the weak-coupling calculations. For example, the fermion gap in the BCS region can be computed by solving the gap equation as

$$
\Delta_{G} \sim \mu \exp \left(-\frac{\pi^{2}}{g} \alpha_{G}\right)
$$

where $G$ denotes the gauge group $\mathrm{SU}\left(N_{c}\right), \mathrm{SO}\left(2 N_{c}\right)$, or $\mathrm{Sp}(2 N), \alpha_{G}$ is some factor involving $N_{c}$ (see [6] for the expression), and we set $g_{\mathrm{SU}}=g_{\mathrm{SO}}=g_{\mathrm{Sp}} \equiv g$. We find the ratios of $\alpha_{G}$ between QCD at large $\mu_{I}$ and $\mathrm{SO}\left(2 N_{c}\right)$ and $\operatorname{Sp}(2 N)$ gauge theories at large $\mu_{B}$ [6],

$$
\begin{aligned}
& \frac{\alpha_{\mathrm{SO}}}{\alpha_{\mathrm{SU}}}=\sqrt{\frac{2\left(N_{c}^{2}-1\right)}{N_{c}\left(2 N_{c}-1\right)}}=\left\{\begin{array}{cc}
1.033 & \left(N_{c}=3\right) \\
1 & \left(N_{c}=\infty\right)
\end{array},\right. \\
& \frac{\alpha_{\mathrm{Sp}}}{\alpha_{\mathrm{SU}}}=\sqrt{\frac{2\left(N_{c}^{2}-1\right)}{N_{c}\left(2 N_{c}+1\right)}}=\left\{\begin{array}{cc}
0.873 & \left(N_{c}=3\right) \\
1 & \left(N_{c}=\infty\right)
\end{array} .\right.
\end{aligned}
$$

Not only these ratios are unity in the large- $N_{c}$ limit as predicted by the orbifold equivalence, but also they are close to unity even for three-colors. On the other hand, the BCS gap in QCD at large $\mu_{B}$ vanishes, and there is no equivalence with this theory in this region [6]. This is not unexpected, because the discrete symmetry used for the projection onto QCD at $\mu_{B} \neq 0$ is spontaneously broken inside the BEC-BCS crossover region of $\mathrm{SO}\left(2 N_{c}\right)$ and $\mathrm{Sp}\left(2 N_{c}\right)$ gauge theories, and the orbifold equivalence should break down as explained in Sec. 3 .

It is also possible to check the universality within effective models and effective theories of QCD and QCD-like theories. For example, one can show that phase diagrams of chiral random matrix models between all the universality classes are universal [6]. An equivalence of phase diagrams between chiral unitary matrix model at $\mu_{B} \neq 0$ and that at $\mu_{I} \neq 0$ outside the BECBCS crossover region was first pointed out in [17] without using the orbifold equivalence. An equivalence between QCD and $\mathrm{SO}\left(2 N_{c}\right)$ gauge theory at small $\mu_{B}$ was also confirmed at the level of chiral perturbation theories [5]. The generalization to all the class of theories in Fig. 1 at any quark density (at $T=0$ ) should be possible, based on the effective field theories recently constructed in [13].

From these nontrivial tests, we expect that the universality might work as an approximate notion in real QCD. 


\section{Conclusion}

We have discussed the universality of phase diagrams in QCD and QCD-like theories through the large- $N_{c}$ orbifold equivalence, which may be valid approximately in three-color QCD. The proposed universality provides a way to evade the sign problem in lattice QCD simulations at $\mu_{B} \neq$ 0, e.g., for the physics related to the chiral transition. Most recently it was rigorously shown that chiral critical phenomena, especially the QCD critical point [18], are ruled out in QCD at $\mu_{B} \neq 0$ where the universality holds at large $N_{c}$ [19]. It would be still important to understand the fate of the chiral transition at $\mu_{B} \neq 0$. The lattice simulations in $\mathrm{QCD}$ at $\mu_{I} \neq 0$ were already performed, e.g., in [20, 21]. In our opinion, further investigations in this direction should be required.

\section{References}

[1] D. T. Son and M. A. Stephanov, Phys. Rev. Lett. 86, 592 (2001) [arXiv:hep-ph/0005225].

[2] J. B. Kogut, M. A. Stephanov and D. Toublan, Phys. Lett. B 464, 183 (1999) [arXiv:hep-ph/9906346].

[3] J. B. Kogut, M. A. Stephanov, D. Toublan, J. J. M. Verbaarschot and A. Zhitnitsky, Nucl. Phys. B 582, 477 (2000) [arXiv:hep-ph/0001171].

[4] A. Cherman, M. Hanada and D. Robles-Llana, Phys. Rev. Lett. 106, 091603 (2011) [arXiv:1009.1623 [hep-th]].

[5] A. Cherman and B. C. Tiburzi, JHEP 1106, 034 (2011) [arXiv:1103.1639 [hep-th]].

[6] M. Hanada and N. Yamamoto, arXiv:1103.5480 [hep-ph].

[7] K. Fukushima, D. E. Kharzeev and H. J. Warringa, Phys. Rev. D78, 074033 (2008). [arXiv:0808.3382 [hep-ph]].

[8] S. Kachru and E. Silverstein, Phys. Rev. Lett. 80, 4855 (1998) [arXiv:hep-th/9802183].

[9] M. Bershadsky and A. Johansen, Nucl. Phys. B 536, 141 (1998) [arXiv:hep-th/9803249].

[10] P. Kovtun, M. Ünsal and L. G. Yaffe, JHEP 0312, 034 (2003) [arXiv:hep-th/0311098].

[11] P. Kovtun, M. Ünsal and L. G. Yaffe, JHEP 0507, 008 (2005) [arXiv:hep-th/0411177].

[12] M. Ünsal and L. G. Yaffe, Phys. Rev. D74, 105019 (2006) [arXiv:hep-th/0608180].

[13] T. Kanazawa, T. Wettig and N. Yamamoto, [arXiv:1110.5858 [hep-ph]].

[14] M. N. Chernodub and A. S. Nedelin, Phys. Rev. D83, 105008 (2011). [arXiv:1102.0188 [hep-ph]].

[15] M. Ruggieri, Phys. Rev. D84, 014011 (2011). [arXiv:1103.6186 [hep-ph]].

[16] R. Gatto and M. Ruggieri, [arXiv:1110.4904 [hep-ph]].

[17] B. Klein, D. Toublan and J. J. M. Verbaarschot, Phys. Rev. D68, 014009 (2003) [arXiv:hep-ph/0301143].

[18] M. A. Stephanov, Prog. Theor. Phys. Suppl. 153, 139 (2004) [arXiv:hep-ph/0402115].

[19] Y. Hidaka and N. Yamamoto, [arXiv:1110.3044 [hep-ph]].

[20] P. de Forcrand, M. A. Stephanov and U. Wenger, PoS LAT2007, 237 (2007) [arXiv:0711.0023 [hep-lat]].

[21] J. B. Kogut and D. K. Sinclair, Phys. Rev. D77, 114503 (2008) [arXiv:0712.2625 [hep-lat]]. 